The Annual of Animal Psychology 12 (1962)

\title{
REVERSAL RETARDATION THROUGH INTRODUCING FORCED NON-REWARDING TRIALS IN OVERTRAINING BY WHITE RATS
}

\author{
JUNJI KOMAKI* \\ Kyoto University
}

The present study was designed for the purpose to evaluate the contribution of continuous rewarding trials as a possible basis of the overlearning effect (the facilitation of discrimination reversal by overlearning).

In ordinary overlearning studies, overlearning $S$ s were given, after original criterion was attained, a definite number of additional overlearning trials and subsequently learned the reversal. Because the Ss rarely made errors on the overlearning trials (especially on the later trials), this treatment unavoidably introduced the successive, extended occurrences of rewarding trials just prior to the beginning of reversal learning. It was highly probable, therefore, that when preceded by the continued series of rewardings the non-rewarding trials in the subsequent reversal might be more effective in attenuating the prior correct response and that the facilitation of reversal (Reid, 10 ; Pubols, 9; Capaldi and Stevenson, 2) was merely due to the faster extinction of the prior response in the overlearning $\mathrm{Ss}$.

According to this interpretation of the overlearning effect, it was expected that, increasing the occurrences of non-rewarding trials during overtraining, the extinction of the original response would be progressively prolonged and the reversal retarded, since the amount of decrement per reversal non-rewarding trial could be assumed to be increasingly less. In the present study, the number of forced non-rewarding trials of incorrect response which were interspersed among forced rewarding trials of correct response in

$\approx$ The writer was greatly indebted to Assistant Professor Ryoji Motoyoshi for his kind suggestions and criticism throughout the study. 
overtraining was varied and it was examined whether or not the reversal learning of a position discrimination would be increasingly retarded as a function of the forced non-rewarding trials involved.

\section{Method}

Subjects: Forty experimentally naive, male albinos were used as $S$ s. At the start of experiment, they weighed from 200 to $240 \mathrm{grm}$. and were from 65 to 75 days old. Three out of them were discarded in the course of runway training, and three female albinos of similar age and weight were added in place of them.

Ss were housed in their individual home cages one week prior to pre-training, and once-a-day food regimen was administered. Water bottle was always available. $15 \mathrm{grm}$. of Solid Food for Rat and Mouse, manufactured by Oriental Yeast Co., Ltd, was determined for their daily ration. It was fed, during experimentation throughout, at varied intervals from the time of last feeding which were rotated within a maximum of 28 hours and a minimum of 20 for every several days. This amount of ration resulted some weight gain in most $S$ s at the end of the experiment.

Apparatus: Apparatus consisted of a set of four straight runways which were used exclusively in pre-training and an alley Y-maze. The Y-maze was $4 \mathrm{in}$. high and, except for goal boxes, $4 \mathrm{in.}$ wide, and had a hardware cloth floor and was covered with hinged hardware cloth ceilings. The length of start box, stem and arm was 7, 18 and $18 \mathrm{in}$., respectively. The goalboxes were $9 \mathrm{in}$. wide and $7 \mathrm{in}$. long and, on the near walls of their respective sides, there were metal fittings to which a stainless steel food-cup could be held.

The maze was painted mid-grey inside and was situated in the center of a wooden flame enclosure of $6 \times 6 \times 6 \mathrm{ft}$. Above the center of the maze, was suspended one rod-shaped electric bulb of $60 \mathrm{w}$., the brightness of which was about 45 lux, when measured at the point of bifurcation. 
Pre-training: On the preceding 4 days to the runway training, $S$ s were handled daily for 3 minutes. On the last of these days, about $2 / 3$ of them ate directly from $E^{\prime}$ s hand, while being gentled. For the following 3 days, $S$ s were given 6 trials of runway training. They were placed on one end of the runway and reached food pellets on the other.

On the eighth day, position preference test was administered. In both goal boxes of the Y-maze, were placed two food pellets, each of which was a solid mixture of milk and flour, cyrindrical in form, and weighed $.05 \mathrm{grm}$. Body length entry beyond either one of the choice doors was determined as choice, and the door was lowered manually to prevent retracing. Ss chose five times and, because they were run in a squad of 3 or 4 , intertrial interval was kept within a range of 3 minutes to 4 .

Experimental procedure: Training consisted of original position discrimination and its reversal and, for overtraining $S$ s, a series of forced overtraining trials between the original and the reversal. Ss were run 10 trials a day, and in the original and reversal non-correction method was utilized.

$S$ was placed in the start box and $5 \mathrm{sec}$. later the start door was raised manually. It was kept in the goal box for $10 \mathrm{sec}$, irrespective of whether it was baited or not. Because $S$ s were trained in a squad of 3 or 4 , intertrial intervals were held within a range of 2 minutes to 3 . A choice was body length entry beyond either choice door, and the doors (start, choice and goal) were lowered manually when $S$ passed below each of them to prevent retracing.

Learning criterion was more than 18 correct responses out of two consecutive days with the restriction of less than one error on the second day. The correct side in the original learning was determined as the non-preferred one.

Upon reaching the criterion, $S$ s were assigned into one of the five groups below so as to be matched in terms of the trials and the correct side in the original learning. 
Control group (7 males and 1 female): Immediately the day after the original criterion was attained, the reversal discrimination was initiated in this group.

100 : 0 group ( 7 males and 1 female) : Prior to the reversal, $S \mathrm{~s}$ were given 100 trials of the forced rewarding trial for the correct response ( $\mathrm{RC}$ trial) for 10 days. In the course of this training, the incorrect choice door was kept closed.

100 : 50 group (7 males and 1 female) : In this group, $100 \mathrm{RC}$ trials and 50 trials of the forced non-rewarding trial for the incorrect response (NI trial) preceded to the reversal. They were arranged so that $20 \mathrm{RC}$ and $10 \mathrm{NI}$ trials composed the trials of every three days.

100 : 100 group ( 8 males) : $100 \mathrm{RC}$ and $100 \mathrm{NI}$ trials were run for 20 days by this group, daily trials being composed of $5 \mathrm{RC}$ and 5 NI trials.

100 : 200 group (8 males) : $100 \mathrm{RC}$ and $200 \mathrm{NI}$ trials over 30 days were overtraining for this group. And the trials for every three days were $10 \mathrm{RC}$ and $20 \mathrm{NI}$ trials.

For each of the last three groups above, arrangement of $\mathrm{RC}$ and NI trials had been predetermined over 30 trials, and it was repeatedly used, being rotated every 3 days. In fact, each of the groups was further divided into two subgroups that were distinguished in terms of whether the last trial of their daily runs was always $\mathrm{RC}$ trial or NI. Because this treatment did not yield any appreciably systematic variation, it was omitted in the data analysis.

The time elapsed from raising of the start door to $S$ 's entrance to either one of the goal boxes or to the particular, forced goal was measured to the nearest half second by means of a stop-watch. And, when on NI trials $S$ refused to enter into the forced arm or to the goal box (after having got into the forced arm) within 2 minutes, $E$ gently guided it by hand.

Correct goal box was baited with 2 grains of pellets depicted above, and in order to minimize secondary reinforcement, food-cup was always removed from incorrect goal box. 


\section{Results}

Means and SD's for the number of trials until criterion in the original and the reversal learning are illustrated in Table 1 for the five groups.

Table 1. Means and $\mathrm{SD}^{\prime} \mathrm{s}$ for the number of trials to reach criterion in the original and the reversal learning

Original

\begin{tabular}{lcccr} 
& Mean & SD & Mean & \multicolumn{1}{c}{ SD } \\
\hline Control & 7.5 & 6.61 & 13.75 & 6.96 \\
$100: 0$ & 7.5 & 6.61 & 21.25 & 10.54 \\
$100: 50$ & 8.75 & 7.81 & 30.00 & 10.00 \\
$100: 100$ & 7.5 & 9.68 & 25.00 & 10.00 \\
$100: 200$ & 7.5 & 6.61 & 25.00 & 11.18
\end{tabular}

A one-way analysis of variance, when applied to the trials in the original, yielded an $F$ of $.038(d f=4,35)$ which was far from the level of significance. Then, these groups can be evaluated to be homogeneous. When a like $F$ test was applied to the trials in the reversal, the differences among them proved to be significant ( $F=$ $2.65 ; d f=4,35 ; p<.05)$. In the separate, pairwise $t$ tests which were applied to the group means, Control group emerged significantly superior to $100: 50$ group $(t=3.53 ; d f=14 ; p<.01)$, to $100: 100$ group $(t=2.44 ; d f=14 ; p<.05)$, and to $100: 200$ group $(t=2.26$; $d f=14 ; p<.05)$. But, the remaining group differences fell short of significance.

As inspected in Table 1, reversed position discrimination was greatly retarded by preceding learning. To evaluate the inhibiting effect several kinds of measures in the reversal learning were rendered to analysis. The first was the number of consecutive errors until the first correct response, and means and $\mathrm{SD}$ 's for the scores are summarized for the five groups in Table 2. $H$ test was applied, but the difference among them failed to reach the level of significance $(H=8.19 ; d f=4 ; p>.05)$. 
Table 2. Means and SD's for the number of the consecutive errors until the first correct response in the reversal learning.

\begin{tabular}{lrc} 
& Mean & SD \\
\hline Control & 6.00 & 2.50 \\
$100: 0$ & 7.86 & 6.03 \\
$100: 50$ & 13.63 & 7.37 \\
$100: 100$ & 6.50 & 3.00 \\
$100: 200$ & 7.88 & 2.26 \\
\hline
\end{tabular}

The second was the number of days when $S$ committed more than 8 errors out of daily 10 trials. Means and SD's for the five groups are illustrated in Table 3. An analysis of variance in one-way classification yielded a significant $F$ value $(F=5.23 ; d f=4,35 ; p<$ .01). And, through pairwise comparison $100: 50$ group proved to be significantly more resistant to extinction than the other 4 groups. However, all the other differences between group means fell short of significance.

Table 3. Means and $S D$ 's for the number of the days when $S$ committed more than 8 errors out of daily 10 trials in the reversal learning.

\begin{tabular}{lrr} 
& Mean & SD \\
\hline Control & .625 & .48 \\
$100: 0$ & .500 & .71 \\
$100: 50$ & 1.750 & 1.09 \\
$100: 100$ & 1.000 & .50 \\
$100: 200$ & .625 & .48 \\
\hline
\end{tabular}

Running times from the opening of the start door to the entrance into either the correct goal or the incorrect in the reversal learning were transformed into reciprocals. And, group $M d n$ 's of the individual $M d n$ response speed for the first three correct responses and those of the individual $M d n$ response speed for the first three incorrect responses are summarized in Table 4.

As for the correct response speed, group $M d n$ 's were significantly different $(H=19.7 ; d f=4 ; p<.01)$. By the use of $U$ tests, the 
Table 4. Group $M d n$ ' $\mathrm{s}$ of the individual $M d n$ response speed for the first three correct and for the first three incorrect responses in the reversal learning.

\begin{tabular}{lcc} 
& Correct & Incorrect \\
\hline Control & 0.343 & 0.584 \\
$100: 0$ & 0.400 & 1.000 \\
$100: 50$ & 0.065 & 0.667 \\
$100: 100$ & 0.098 & 0.500 \\
$100: 200$ & 0.100 & 0.236
\end{tabular}

three NI groups (groups $100: 50,100: 100$ and $100: 200$ ) proved to be slower in correct responding than Control group and than $100: 0$ group $(p \leqq .01)$. However, none of the differences between Control and 100:0 groups and between the three NI groups reached the significant level of confidence.

On the other hand, incorrect response speed to the prior positive side was fairly systematically varied. As inspected in Table 4, speed of running was progressively lowered as the number of NI trials was increased. When an $H$ test was applied, a value of 17.8 yielded which was significant beyond the $1 \%$ level of confidence $(d f=4)$. Making use of pairwise $U$ tests, 100:200 group proved to be significantly slower than the other 4 groups, and 100: 100 group did than 100:50 group ( $U$ value between 100:0 and 100:100 groups fell within the range between the $10 \%$ and the $5 \%$ level of confidence).

In the present, experimental set-up, overtraining by forced choice method was administered after the attainment of original criterion. To examine whether or not overtraining $S$ s still differentially performed between on $\mathrm{RC}$ and on NI trials, daily response speed in the course of the overtraining was rendered to statistical analysis for each $S$ of the three NI groups. Although on the initial few days in the overtraining significant differences in speed were not obtained in some $\mathrm{Ss}$, on the fifth day and thereafter more than $7 \mathrm{Ss}$ of each group $(N=8)$ emerged to run significantly faster on $\mathrm{RC}$ trials than on NI trials ( $U$ tests were applied). 


\section{Discussion}

Unfortunately, the present study failed to replicate the overlearning effect ( $c f$. Pubols, 9). Although statistically unreliable, 100: 0 group was inversely inferior to Control group in terms of trials to the reversal criterion. Therefore, the present study does not serve as a direct test of the hypothesis that continuous occurrences of rewarding trials in overtraining lead to faster extinction of the original response and that the overlearning effect is merely due to the faster extinction.

Reversal learning was significantly retarded for the three NI groups. However, the introduction of non-rewarding trials by means of forced choice technique turned out to be a source of complication. In view of the significantly lower correct response speed for the NI groups in the reversal, the NI trials during overtraining seem to have increased the avoidance tendency ( $c f$. D' Amato and JAGODA, 3 ) or inhibition of the prior incorrect side beyond the degree in the original learning. Therefore, it seems to be undetermined whether the observed retardation of reversal for the NI groups is due to the increased inhibition or to the differential speed of extinction.

Because the three NI groups were nearly equal similarly with respect to NI trial speed at the end of overtraining, 50 NI trials appear to be sufficient to accumulate the inhibition to the asymptotic level. However, the strength of the prior correct response itself seems to be greatly affected by the increase in NI trials. As shown in Table 4, the incorrect response speed was progressively lowered as the increase in NI trials. In other words, the prior correct response was the most firmly established in 100:50 group and the least in $100: 200$ group. The differences for the strength of the prior correct response are presumably the reason for the results that extinction was especially prolonged in 100:50 group but not in groups $100: 100$ and $100: 200$. On the other hand, $100: 0$ group, despite its greatest speed on the incorrect responding in the reversal, 
was less resistant to extinction than 100:50 group (cf. Tables 3 and 4). It suggests that the extinction of prior correct response proceeded relatively faster in 100: 0 group. Although partly attributable to the relatively weak inhibition of the prior incorrect side, it also seems to indicate that non-rewarding trials in the reversal might be more effective with respect to the extinction of $100: 0$ group.

It should be noted that the effect of reversal facilitation seems to be more unstable to appear in positional discrimination than in non-positional discrimination learning. As for the latter, all recent studies obtained the effect (NorTh and Clayton, 8; SASAKI, 11; Brookshire et al ., 1; D' Amato and Jagoda, 3 ; Komaki, 6). And, in the case of position discrimination, TAKEMUra (12) and Brookshire et al. (1) obtained the effect, but Iwahara (7), KomaKi (5) and D' Amato and JAgoda (4) failed.** The discrepancies in results between the two learning situations seem suggestive in considering the effect of overlearning and need to be examined in future experimentation.

\section{Summary}

In order to evaluate the effect of continued rewarding trials on the discrimination reversal, 5 groups of albino rats $(N=8)$ learned position discrimination in an alley Y-maze. Four overtraining groups received differential treatments in the period of overtraining. 100:0 group was forced to the correct side over 100 trials, and the other three (groups 100:50, 100:100 and 100:200) were forced to the correct side for 100 trials and to the incorrect for 50, 100 and 200 trials, respectively.

The effect of reversal facilitation was not obtained, and the reversal emerged to be retarded in groups $100: 50,100: 100$ and $100: 200$. And, the extinction of prior correct response was es-

** HiLL and others (Northwestern University) similarly failed to obtain the effect in experiments on a T-maze. 
pecially prolonged in $100: 50$ group.

Non-rewarding trials of incorrect response by means of forced choice method turned out to increase the inhibition of incorrect side beyond the degree in the original learning. Because of this complicating effectiveness of forced non-rewarding trials, the initial attempt was not accomplished.

(Manuscript received June 16,1962)

\section{References}

1. Brookshire, K, H., Warren, J. M., \& Ball, G. G. Reversal and transfer learning following overtraining in rat and chicken. J. comp. physiol. Psychol., 1961, 54, 98-102.

2. Capaldi, E. J., \& Stevenson, H. W. Response reversal following different amount of training. J. comp. physiol. Psychol., 1957, 50, 195-198.

3. D' Амato, M. R., \& Jagoda, H. Analysis of the role of overlearning in discrimination reversal. J. exp. Psychol., 1961, 61, 45-50.

4. D' Амato, M. R., \& JaGoda, H. Overlearning and position reversal. $J$. exp. Psychol., 1962, in press.

5. KомакI, J. Overlearning effect in spatial and brightness discrimination learning by white rats. Proc. the 65th Kansai Psychol. Association Meeting. 1959.

6. Komakr, J. The facilitative effect of overlearning in discrimination learning by white rats. Psychologia, 1961, 4, 28-35.

7. Iwahara, S. On overlearning and shift learning by white rats. Proc. the 23rd JPA Meeting, 1959.

8. North, A. J ., \& Clayton, K. N. Irrelevant stimuli and degree of learning in discrimination learning and reversal. Psychol. Rep., 1959, 5, 405-408.

9. Pubols, B. H. The facilitation of visual and spatial discrimination reversal by overlearning. J. comp. physiol. Psychol., 1956, 49, 243-248.

10. REID, L. S. The development of non-continuity behavior through continuity learning. J. exp. Psychol., 1953, 46, 107-112.

11. SASAKI, M. The effect of overtraining upon transfer in discrimination learning. Proc. the 24th JPA Meeting, 1960, 199-200.

12. TAKemura, $K$. The effect of overlearning on the subsequent task with different type of solution. Proc. the 25th JPA Meeting, 1961, 132. 